\title{
Review of Communication Technologies for Electric Vehicle Charging Management and Coordination
}

\author{
Eiman ElGhanam (D), Mohamed Hassan*(D), Ahmed Osman and Ibtihal Ahmed \\ Department of Electrical Engineering, American University of Sharjah, \\ Sharjah P.O. Box 26666, United Arab Emirates; eelghanam@aus.edu (E.E.); aosmanahmed@aus.edu (A.O.); \\ g00070409@aus.edu (I.A.) \\ * Correspondence: mshassan@aus.edu
}

Citation: ElGhanam, E.; Hassan, M.;

Osman, A.; Ahmed, I. Review of

Communication Technologies for

Electric Vehicle Charging

Management and Coordination.

World Electr. Veh. J. 2021, 12, 92.

https://doi.org/10.3390/

wevj12030092

Academic Editor: Joeri Van Mierlo

Received: 14 June 2021

Accepted: 24 June 2021

Published: 28 June 2021

Publisher's Note: MDPI stays neutral with regard to jurisdictional claims in published maps and institutional affiliations.

Copyright: (c) 2021 by the authors. Licensee MDPI, Basel, Switzerland. This article is an open access article distributed under the terms and conditions of the Creative Commons Attribution (CC BY) license (https:// creativecommons.org/licenses/by/ $4.0 /)$

\begin{abstract}
Recently, electric vehicles (EVs) have been introduced as an alternative method of transportation to help mitigate environmental issues, such as carbon emissions and fuel consumption, caused by conventional transportation systems. The implementation of effective EV charging systems is essential to motivate mass adoption of EVs. Accordingly, fast and reliable communications between the charging systems and the EVs are vital for efficient management of the charging process. Different radio access technologies (RATs) are discussed in the literature to enable communication between the highly mobile EVs and the charging subsystems, to collect and exchange information such as state of charge ( $\mathrm{SoC})$, users' locations, and charging decisions between the different network entities. This information can be used to coordinate charging plans and select the optimal routes for moving vehicles. This paper presents a survey of existing literature on vehicular communications for EV charging coordination and management. The communication requirements and feasible communication technologies for vehicular communication are first discussed in details. A review of the physical layer security strategies is then presented and the role of the different RATs in EV charging coordination and management is described and studied.
\end{abstract}

Keywords: electric vehicles; IoEV; vehicle-to-vehicle (V2V); vehicle-to-infrastructure (V2I); dynamic charging; direct short-range communication (DSRC); C-V2X; NR-V2X; physical layer security; charging coordination; scheduling

\section{Introduction}

The growing concern regarding fossil fuel depletion and environmental pollution significantly impacts transportation systems, thereby leading to the gradual shift from conventional internal combustion engine (ICE)-based vehicles toward more environmentally friendly solutions. Electric vehicles (EVs) have been receiving considerable attention as an eco-friendly alternative transportation solution to reduce air pollution and conserve energy. However, the large-scale adoption of EVs is still limited due to the significantly longer recharging time compared to the refueling time of ICE vehicles, the limited driving range restricted by the $\mathrm{EV}$ battery capacity, and the higher initial investment costs due to the relatively higher EV purchase prices compared to their fuel-operated counterparts [1,2]. In fact, efforts to reduce range anxiety by increasing EV battery capacity further contribute to higher EV purchase prices and discourage mass adoption of EVs [3]. Nevertheless, according to $[3,4]$, the total cost of operation (TCO) of EVs can be lower than that of diesel and gasoline operated vehicles when acknowledging factors such as the higher efficiency of EV motors and the comparatively lower cost of electricity, particularly with the utilization of renewable energy sources and smart grids [5,6]. Furthermore, the increasing deployments of EV charging infrastructures as well as the government subsidies and other incentive-based green energy programs all motivate the adoption of EVs.

Charging an EV can be performed either using a charging cable, where the EV is plugged into a power outlet, or through wireless charging with no cables attached $[2,7,8]$. 
Wired charging requires direct physical connectivity between the EV and the charging socket, thereby imposing an electrocution hazard to the EV user, particularly during harsh weather conditions. To overcome this hazard, effective electrical isolation is required between the EV body, the EV supply equipment (EVSE) and the mains power grid, using high-frequency isolation transformers [3] or other isolation techniques. These transformers, however, are costly to implement and maintain, and they impact the switching operation of the EV chargers. In addition, hardware compatibility between the EV charging connector and the charging inlet point is essential to enable wired EV charging [9]. This impacts the convenience to EV users, as it limits the locations at which an EV can be charged to those compatible and/or interoperable with the EV connector.

In contrast, wireless charging is adopted as an alternative charging method, where no physical connection is required between the EV and the power source. Wireless charging is becoming more common due to its flexibility, convenience and, more importantly, higher safety factor. It is commonly categorized into three modes: static charging, dynamic charging, and quasi-dynamic charging [10,11]. In static charging, energy is transferred from the grid to the EV using inductive wireless power transfer while the EV is parked over a wireless charging pad. A dynamically charged vehicle, on the other hand, receives the charging energy wirelessly during its motion over a set of coils placed on the road $[12,13]$. Quasi-dynamic charging is an integration of the two modes in which the EV is charged during transient stops at intersections and traffic signals [14]. By allowing an EV to receive energy wirelessly during its motion, the range anxiety of EV drivers can be significantly reduced, particularly in cities with large traveling distances between the different locations. Dynamic wireless charging (DWC) also eliminates driving delays due to EV charging downtime [15] and introduces opportunities for EV battery downsizing and EV price reduction $[16,17]$. In addition, the development of vehicular dynamic wireless power transfer (DWPT) solutions reveals opportunities for wireless energy exchange between moving vehicle, using mobile energy disseminators (MEDs) as movable energy supply units $[18,19]$, as well as using direct energy transfer from one passenger vehicle to another (vehicle-to-vehicle (V2V) energy transfer) [20,21].

The integration of the aforementioned EV charging solutions within the city infrastructure is expected to increase EV adoption by ensuring ubiquitous charge availability and improving the convenience levels for EV owners. This contributes to the establishment of an internet of electric vehicles (IoEV) in which EVs are considered as intelligent entities equipped with multiple on-board sensors and actuators, and can communicate and exchange information securely with one another and with the surrounding infrastructure to enable driving and charging coordination, energy management, and traffic optimization $[22,23]$. A smart city infrastructure model demonstrating the integration of different EV charging infrastructures in an IoEV is shown in Figure 1.

In order to enable effective EV charging coordination and management in an IoEV, effective communication strategies need to be implemented, in which a communication link is established between the $\mathrm{EV}$ and the charging infrastructure, i.e., vehicle-to-infrastructure (V2I) and infrastructure-to-vehicle (I2V) communication, as well as between neighboring EVs, i.e., vehicle-to-vehicle (V2V) communication. This is because the lack of communication and coordination between EVs within the city infrastructure is expected to cause congestion, inefficient energy distribution, imbalanced loads, and other adverse effects on the operation of the grid. In contrast, smart charging coordination, involving massive data exchange between the different network entities, is expected to solve these issues by utilizing advanced information and communication technologies (ICT) to build a communication infrastructure that enables load balancing, charge scheduling, and reliable authentication and billing services [24,25].

Different communication link characteristics are essential to address the requirements of the different charging modes. For example, a static charging scenario requires a high security and high reliability communication link to ensure accurate information exchange between the EV and the EVSE, but can be tolerant to a certain delay since the EV is 
stationary [26,27]. Dynamic wireless charging, on the other hand, is intolerant to delay and requires a very low latency and high-reliability, two-way communication link to enable effective communication with the charging infrastructure during the vehicle's mobility on the road [28]. The capacity of the communication link is another important characteristic that depends on the volume of the information to be exchanged. The nature of this information is also a factor as it may include higher priority messages, such as safety messages and emergency calls, and lower priority messages including information on the EV's current state of charge ( $\mathrm{SoC})$, desired charging power level, etc. In general, the required communication systems for enabling effective EV wireless charging must provide support for different data types and priorities and must offer the ability to allocate different quality levels to each while ensuring interoperability [24]. Furthermore, the mobility of interconnected vehicles is expected to cause fast channel fading and other channel impairments due to the high speeds of the vehicles. For time-critical applications, such as dynamic wireless charging of EVs, the proposed communication system needs to handle two-way communications at a high speed in real-time with ultra-low latency while enabling handovers between the different communication domains and the different elements of the charging infrastructure.

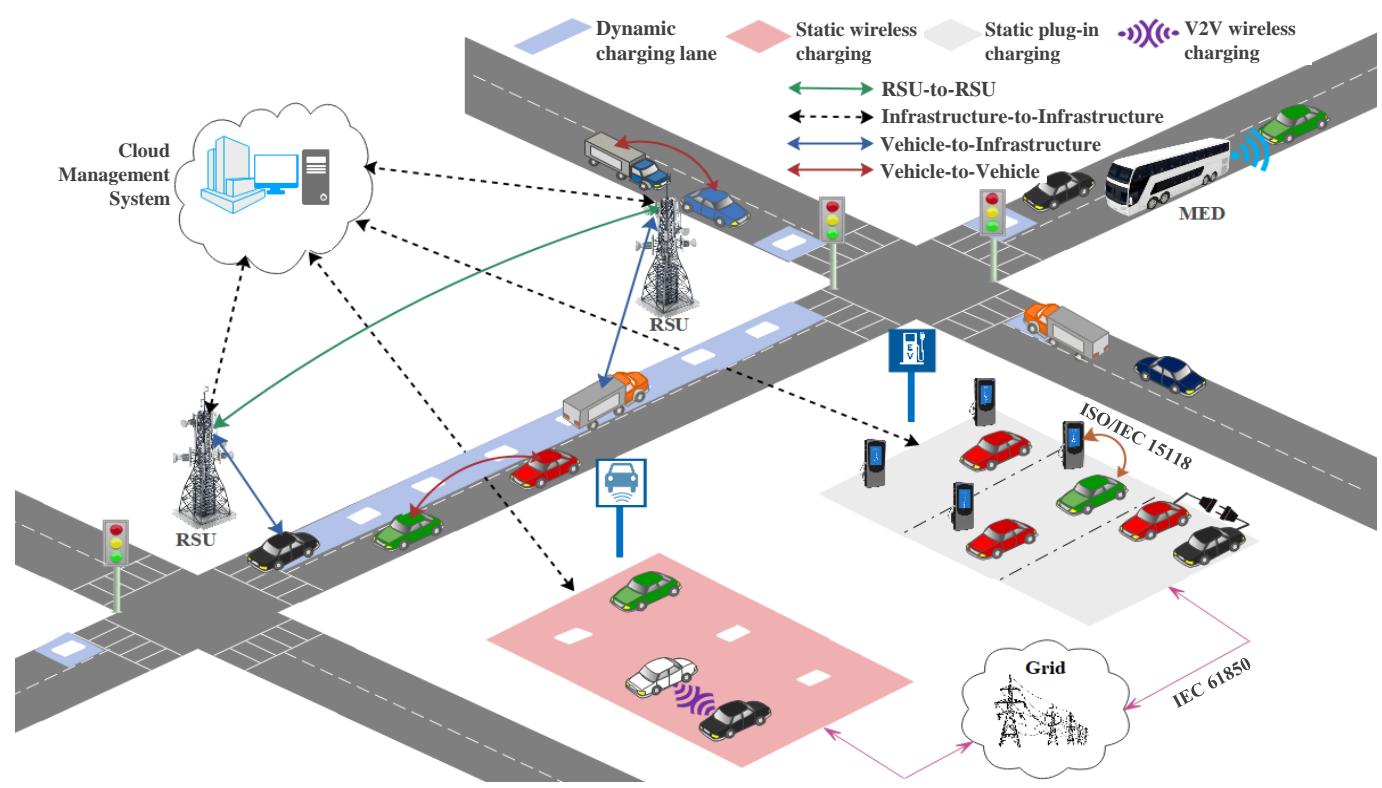

Figure 1. Overview of EV charging solutions in an IoEV within a smart city infrastructure.

Accordingly, a heterogeneous network is required with multiple radio access networks to enable effective communication between various network entities in a large-scale EV charging infrastructure that incorporates static, dynamic, and quasi-dynamic charging [29]. This work aims to survey existing literature on the different communication technologies that enable effective EV charging coordination and management. In particular, Section 2 of this paper presents an overview of the different radio access technologies (RAT) that address the requirements of vehicular communications. A study of the utilization of some of these technologies for reliable information exchange during wireless EV charging is presented in Sections 3-5. In Section 6, the paper surveys the different physical layer security techniques proposed in the literature to establish secure vehicular communication links. Ultimately, the paper highlights how these different technologies can be utilized for effective EV charging coordination and management, in Section 7. The paper is then concluded in Section 8.

\section{Vehicular Radio Access Technologies}

Different technologies have been proposed in the literature to support communication between EVs and the different elements of the infrastructure [30]. Direct wired commu- 
nication and message exchange between the EVs and EVSEs are currently established based on the IEC 61850 and ISO/IEC 15118 standards [23,30]. In addition, high-speed broadband communications over low-voltage power lines are reported in [31,32], in which the same charging cable is utilized to simultaneously carry communications signals along with the charging power. This power line communication (PLC) system demonstrated a theoretical maximum data rate of $14 \mathrm{Mbps}$ which is significantly higher than the $100 \mathrm{kbps}$ required bandwidth estimated by the US Department of Energy for enabling at-home EV charging [26]. Nevertheless, while wired communication solutions offer high security, high reliability, and a sufficiently high data rate, they can only be utilized for plug-in and static wireless charging systems. They cannot, however, be used for DWC systems due to the mobility of the EVs. Furthermore, low data rate and short-range wireless communication systems such as Bluetooth and ZigBee can also support vehicular communications in wired and static wireless charging [33-35], but are not appropriate for dynamic charging. This is because, in dynamic charging, micro-charging sessions that last for just a few milliseconds are required, thereby demanding an ultra-low latency solution. Hence, low-latency, high-reliability, and wide-range wireless communication technologies need to be utilized for V2I, I2V, and V2V communications during dynamic wireless EV charging. A detailed review of, and comparison between, the potential of different wireless communication technologies for EV charging management is presented in this work.

Vehicular communication solutions are inevitable enablers for wireless EV charging system management as they enable the information exchange required for charge scheduling, coordination, vehicle routing, authentication, and billing. For instance, an EV requesting the charging service has to send a charging request with the EV information including EV speed, current location, destination, maximum battery capacity, initial SoC and desired final SoC. Charging reservation information is also exchanged together with periodic real-time updates on arrival time, expected charging time and location, and charging prices. A sample message exchange sequence for charging coordination and reservation between EVs and a stationary charging station (CS) through a global aggregator (GA) is presented in [36], as shown in Figure 2.

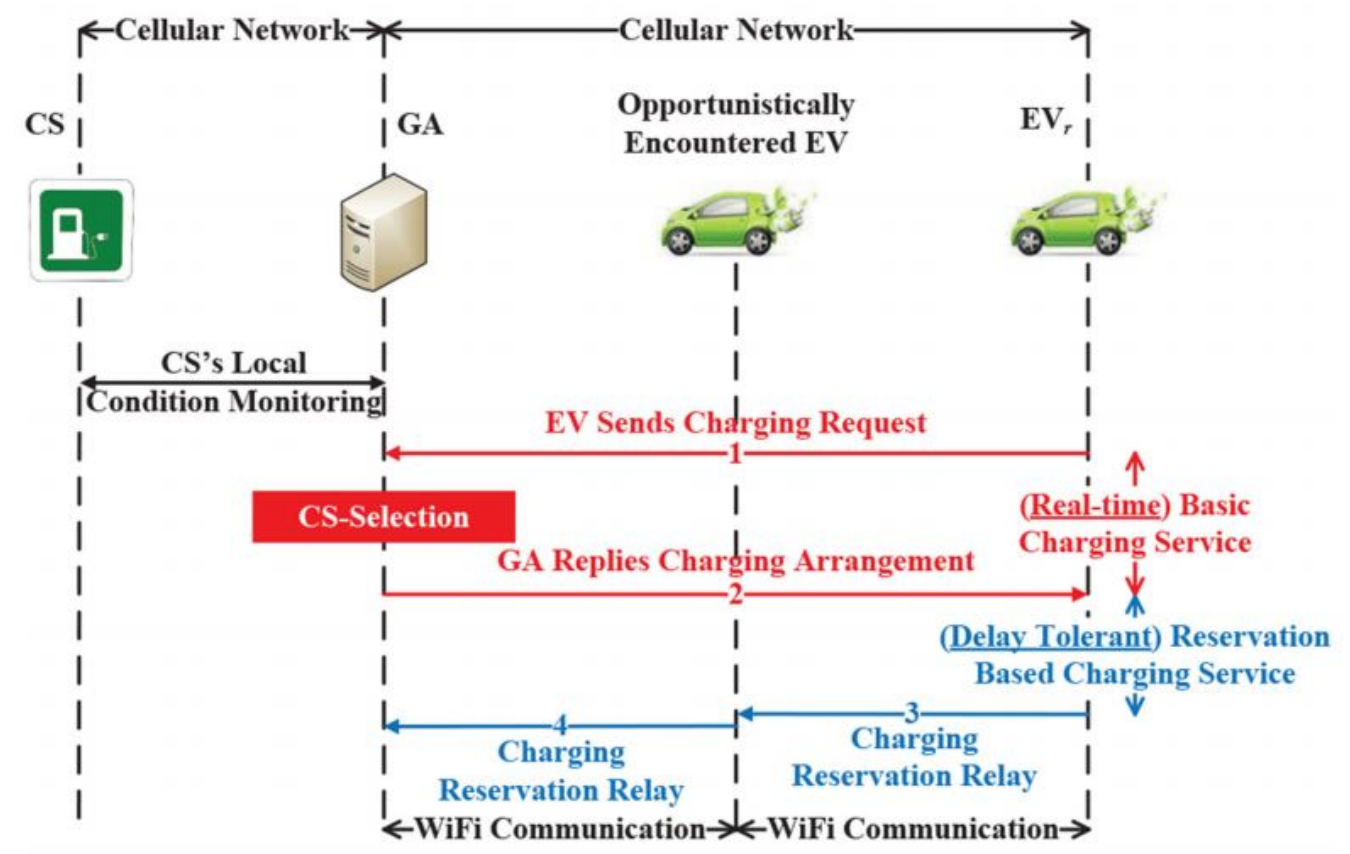

Figure 2. A sample message exchange sequence for an EV charging coordination and reservation system. (02021 IEEE. Reprinted, with permission, from [36]. 
As observed in Figure 2, wireless communication technologies, in this case cellular networks and Wi-Fi communications, are required to enable information exchange between the EVs requesting charge and the charging infrastructure. The choice of the most suitable communication channel and radio interface impacts the speed, security and reliability of the coordination of the charging process. The performance requirements for a vehicular radio access network to be suitable for EV charging management can be summarized as follows:

- Latency: While the end-to-end communication latency requirement for at-home static EV charging is estimated by the US Department of Energy to be around $2 \mathrm{~s}$ [26], this value needs to be significantly smaller for dynamic charging, although a specific reference value is not yet defined by the standards. Dynamic wireless EV charging is a time-sensitive, delay-intolerant service given the short lane-crossing duration at typical EV driving speeds [12]. Moreover, vehicular communication networks need to include congestion detection and prevention mechanisms to avoid undesirable delays [37].

- Throughput: Based on the 2010 US Department of Energy report [26,32], the throughput required for home EV charging systems can vary between 10 and $100 \mathrm{kbps}$. Nevertheless, most of the current vehicular applications are bandwidth-hungry and demand a significantly higher data rate [38]. Since the communication link is expected to carry charging-related messages as well as other information in variable-sized packets, the higher the throughput, the more effective the communication link.

- Reliability: This is a crucial metric for the coordination and management of EV charging services since charging decisions require robust, uninterrupted data exchange with ubiquitous coverage and quality-of-service (QoS) guarantees, i.e., high reliability is required. Reliability is also particularly important for safety-critical applications and is hence an important factor in the selection of effective charge-enabling vehicular communication networks. The reliability of the communication channel is also an important factor in vehicular communications due to the expected channel fading and other impairments during vehicular mobility [39].

- Security and Privacy: One of the most critical requirements in vehicular communications is the security and privacy of the information shared between the EVs and the other network entities. An end-to-end secure data transfer is essential to provide protection against unauthorized transactions and other potential attacks [40-43]. In addition, privacy-sensitive data, such as EV location, EV ID, and payment information, must be protected against potential misuse by other network entities [44].

With these performance requirements in mind, different radio access technologies (RATs) are proposed in the literature for wireless vehicular communications, including cellular networks (3G, 4G, and 5G), direct short-range communication (DSRC), and WiFi [45]. A general comparison between the specifications of these RATs is reported in Table 1.

Table 1. Comparison between some wireless communication technologies for on-the-road vehicular communications [46,47].

\begin{tabular}{|c|c|c|c|c|c|}
\hline Feature & DSRC & 3G-UMTS & 4G-LTE & $\begin{array}{l}5 G^{*} \\
\text { Sub-6 GHz }\end{array}$ & Wi-Fi \\
\hline Coverage & $1 \mathrm{~km}$ & $10 \mathrm{~km}$ & $30 \mathrm{~km}$ & $1-2 \mathrm{~km}$ & $100 \mathrm{~m}$ \\
\hline Max. throughput & 3-27 Mbps & $2 \mathrm{Mbps}$ & $300 \mathrm{Mbps} * *$ & $2.4 \mathrm{Gbps}^{* *}$ & 6-54 Mbps \\
\hline Mobility support & Medium & High & Very high & Ultra high & Low \\
\hline Bandwidth & $10 \mathrm{MHz}$ & $5 \mathrm{MHz}$ & 1.4-20 MHz & 5-100 MHz & $20 \mathrm{MHz}$ \\
\hline Spectrum & Licensed & Licensed & Licensed & Licensed & Unlicensed \\
\hline
\end{tabular}


The different RATs presented in Table 1 vary greatly in terms of their throughput and coverage ranges as well as in their support for user mobility, which impacts their utilization in vehicular communications. Accordingly, several comparative studies are reported in the literature, comparing the performance of these technologies in V2V and V2I communications. The authors in [48] study the feasibility of vehicular communications using digital broadcasting, cellular (3G-UMTS), and DSRC technologies, based on coverage and capacity requirements. In their work, the authors illustrate that UMTS and DSRC technologies have extended their capabilities to support V2V and V2I communications, while the digital broadcast technology is shown to be capacity limited and can only accommodate a small number of users.

A comparison between different communication technologies is also presented in [24] including cellular, DSRC, FM radio, Wi-Fi, and WiMAX. However, due to the high latency of WiMAX and the limited coverage and capacity of $\mathrm{Wi}-\mathrm{Fi}$, the most feasible schemes for vehicular communication are highlighted by the authors in [24] to be cellular technologies and DSRC. The authors in [46] also compare the different characteristics of Wi-Fi to those of cellular and DSRC networks and conclude that the shorter coverage range and lower mobility support of Wi-Fi compared to UMTS, LTE, and DSRC make it difficult to be utilized for information exchange during dynamic charging of mobile EVs [46]. This is because the small Wi-Fi coverage range shortens the dwell-time, and the access point discovery process in the short EV crossing time presents a significant challenge [38]. Furthermore, since $\mathrm{Wi}$-Fi operates in the unlicensed 2.4 and $5 \mathrm{GHz}$ bands, a vehicular communication network based on $\mathrm{Wi}-\mathrm{Fi}$ is more prone to interference, which compromises the security and privacy of the data exchange process.

Another comparative study between DSRC and 4G-LTE for V2V and V2I communications is presented in [49] based on field experiments, without particularly addressing dynamic charging requirements. The reported results reveal that the round trip time (RTT) of a traffic text message broadcast using DSRC is around 10 milliseconds over a range of vehicle traveling velocities between 30 and $120 \mathrm{~km} / \mathrm{h}$. For LTE, on the other hand, the RTT is slightly lower than 100 milliseconds at $30 \mathrm{~km} / \mathrm{h}$ and increases to around 150 milliseconds at $120 \mathrm{~km} / \mathrm{h}$. This means that despite the broad coverage and higher data rates offered by LTE, DSRC offers significantly lower latency and is more suitable for safety-related applications, despite the additional costs of implementation of its roadside units (RSUs). Using LTE for vehicular communications has much lower deployment costs as it utilizes the existing $4 \mathrm{G}$ cellular network infrastructure.

Accordingly, by analyzing the results of the aforementioned studies, it can be concluded that short-range wireless communication technologies, such as Wi-Fi, can only be utilized for stationary EV charging systems, although their limited throughput and questionable security significantly impact their reliability and QoS. They are, however, unsuitable for EV DWC applications due to their coverage and capacity limitations and their weak mobility support. On the other hand, the use of other wide-range RATs, such as DSRC and 4G/5G cellular networks for EV charging applications, needs to be further studied and compared to draw realistic conclusions on their capability to enable and support wireless EV charging systems, particularly in dynamic charging modes.

\section{Dedicated Short-Range Communication (DSRC)}

Dedicated short-range communications (DSRC) is a wireless communication technology designed to support automotive and intelligent transportation system (ITS) applications to enable short-range information exchange between DSRC-enabled devices [50]. In general, there are two communication nodes defined in DSRC: the on-board unit (OBU), also known as the mobile node and is located in the vehicle, and the roadside unit (RSU), or the fixed node, which is installed on the roadside infrastructure. Two physical layer protocols are adopted in DSRC networks, namely IEEE 802.11p and IEEE 802.11bd. 


\subsection{IEEE 802.11p DSRC}

The Institute of Electrical and Electronics Engineers (IEEE) introduced a protocol of 1609 family of standards and called it wireless access in vehicular environments (WAVE) to support DSRC-based operations [51]. Higher layers of the protocol stack are based on the IEEE 1609 series standard, while the physical layer is based on IEEE 802.11p [52]. This is further detailed in Figure 3, showing the two planes constituting the DSRC protocol: the management plane and the data plane.

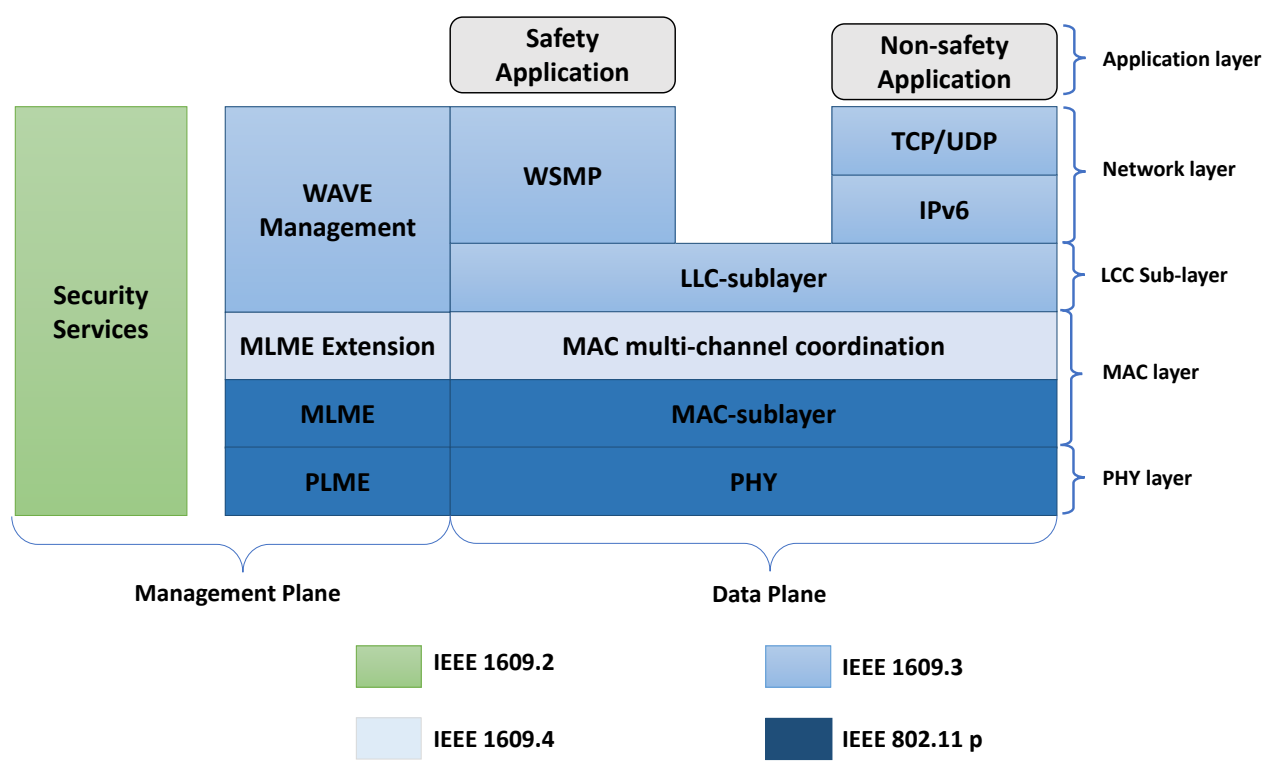

Figure 3. WAVE protocol stack showing management plane and data plane.

DSRC is considered as a viable technology for vehicular communication, addressing both V2I and V2V communications. The Federal Communications Commission (FCC) dedicated $75 \mathrm{MHz}$ of spectrum at the $5.9 \mathrm{GHz}$ band for implementing V2V and V2I communications using DSRC [24,53]. The authors in [54] explain that DSRC is a preferred technology for wireless communication in vehicular networks due to its low latency, high reliability, security, and possible allocation of high priority messages for safety applications. This is also illustrated in Figure 3, showing that the data plane supports two protocols, WAVE short message protocols (WSMP) for high priority and safety applications with low latency, and IPv6 for less critical applications [55].

Despite its advantages in message prioritization and low latency, the short operation range of DSRC technologies based on IEEE 802.11p introduces difficulties in non-line-ofsight (NLOS) operation, which weakens the communication link between the EVs and the RSUs during traffic congestion. In fact, the performance of DSRC rapidly deteriorates in high density traffic networks due to massive packet collisions during simultaneous transmissions [56]. This, accordingly, introduces problems with quasi-dynamic charging coordination, particularly where EVs benefit from high traffic densities to extend their effective charging duration and increase the amount of received energy.

\subsection{IEEE $802.11 b d$ DSRC}

The next generation DSRC technology, namely IEEE 802.11bd DSRC, is introduced to overcome problems with interferences and NLOS operation faced by DSRC based on IEEE 802.11p. In the current version of IEEE 802.11bd, Doppler shifts are counteracted by the introduction of midambles, analogous to preambles but located at the center of the frame, to provide reliable channel estimates with the fast varying channels experienced during vehicle motion [56,57]. In addition, multiple retransmissions are allowed in IEEE $802.11 \mathrm{bd}$, in contrast to IEEE 802.11p, to enhance the reliability of the communication link. Furthermore, physical layer latency, also known as transmission latency within the air 
interface, is smaller for IEEE 802.11bd in comparison with IEEE 802.11p. This is explained by the authors in [58] who clarify that IEEE 802.11bd allows higher order modulation and coding schemes including 256-QAM, which were not utilized in IEEE 802.11p. In addition, four more data carriers are available in $802.11 \mathrm{bd}$. Collectively, these features guarantee better EV charging coordination and management, particularly in dynamic and quasidynamic charging modes where EV energy updates need to be actively communicated to the infrastructure, and charging activation, coordination, and billing messages need to be exchanged with minimal interference to ensure privacy of the exchanged messages.

\subsection{Multiuser Channel Access}

Multiuser channel access in a vehicular network is more challenging due to the highspeed mobility of the vehicles, the frequent topology variations, and the inevitable network resource sharing. In an IoEV in particular, channel access techniques need to guarantee high reliability, collision avoidance, and low computational complexity. The majority of the proposed channel access techniques for vehicular ad hoc networks (VANET) adopted contention-based medium access control (MAC), in which a node tries to access the channel using carrier-sense multiple access (CSMA). Collisions may occur if another node senses the channel and decides to transmit a packet at the same time [59]. To avoid this issue, CSMA with collision avoidance is recommended by IEEE 802.11p standard for DSRC physical layer [60]. In contrast, contention-free channel access is considered by assigning the channel to only one node at any given time [61] using time division multiple access (TDMA), frequency division multiple access (FDMA), and code division multiple access (CDMA). The authors in [61] claim that TDMA is the most appropriate technique compared to other contention-free protocols. Several works discussed hybrid channel access that makes use of the characteristics of the two mechanisms to improve the performance and minimize the collision rate [62-64]. A comparison between contention-based and contention-free MAC protocols is presented in Table 2.

Table 2. Comparison between contention-based and contention-free MAC protocols [61].

\begin{tabular}{lllll}
\hline Feature & Channel Utilization & Collisions & Throughput & Packet Loss \\
\hline $\begin{array}{l}\text { Contention-based } \\
\text { (CSMA/CA) }\end{array}$ & Inefficient & High & Medium & High \\
$\begin{array}{l}\text { Contention-free } \\
\text { (TDMA) }\end{array}$ & Efficient & Low & High & Low \\
\hline
\end{tabular}

\section{Cellular Networks}

In addition to DSRC, cellular networks have been recommended as a communication framework for vehicular communications, especially for V2I communications, considering wide cellular coverage, low latency, high capacity, and infrastructure readiness due to the current massive cellular network deployments worldwide. In fact, the European Telecommunications Standards Institute (ETSI) addressed vehicular communications using a set of standards for intelligent transportation systems (ITS) to identify the different layers of the V2X and I2V communication systems on top of the cellular physical layer [65]. These standards mainly depend on the LTE network infrastructure with certain modifications to enable effective V2X communications, and address the utilization of these vehicular communications in traffic management, emergency management, driving assistance, etc.

\subsection{UMTS and LTE}

The feasibility of a UMTS-based network to support vehicular communication is evaluated in [66], in which a highway scenario is simulated. Results show that the UMTS network can support V2I communications for non-safety-critical applications. Nevertheless, according to the analysis performed by [67], UMTS offers a round trip time of $350 \mathrm{~ms}$ for cooperative awareness messages (CAM) at intersections, which is more than three times 
larger than the $100 \mathrm{~ms}$ offered by LTE. This makes UMTS questionable for safety-related message exchange at traffic intersections at which quasi-dynamic charging is anticipated to be deployed. In general, LTE is envisioned to well-support V2I and I2V communications and is selected as the underlying network for ETSI ITS communication standards [65]. Furthermore, the simulation results reported in [68] demonstrate that LTE enhances the system throughput while maintaining approximately the same cell coverage compared to 3G networks.

Nevertheless, while LTE can meet the requirements of safety services under low and medium traffic scenarios, its performance may experience significant degradation under heavy load conditions [50]. To reduce latency and improve the system throughput, the authors in [69] suggest utilizing LTE femto access points (FAPs) as relay nodes that can be deployed in buses and large trucks to provide better connectivity. Accordingly, the authors divide the spectrum between cellular users and vehicles, instead of sharing resources, to avoid interference and propose a power control algorithm to maximize the total throughput while mitigating the co-channel interference. With the help of low-cost FAPs and the proposed sub-channel power control, the scheme proposed in [69] provides better throughput and queuing delay compared to traditional DSRC vehicular network in IEEE 802.11p. Another approach to improve the latency performance of LTE networks in vehicular communications is to shorten the transmission time interval (TTI) [70], as shown in Figure 4. However, this approach increases the communication overhead due to additional scheduling and retransmissions.

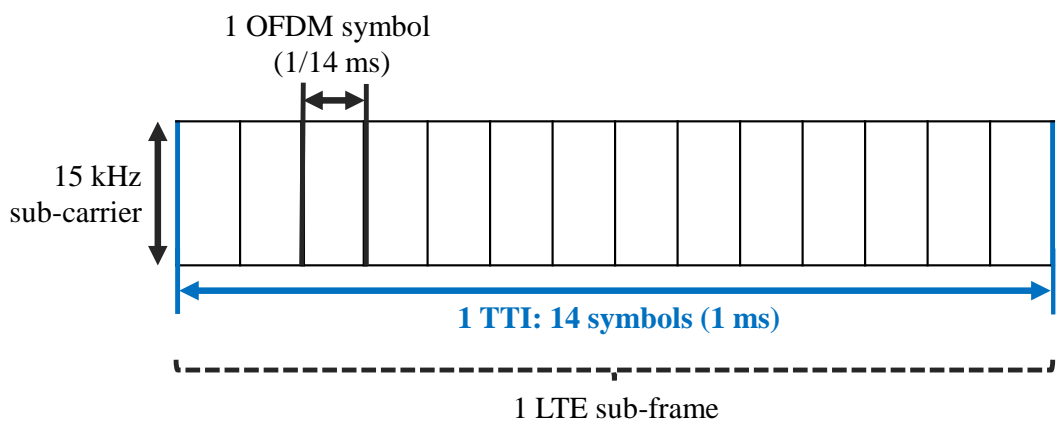

(a)

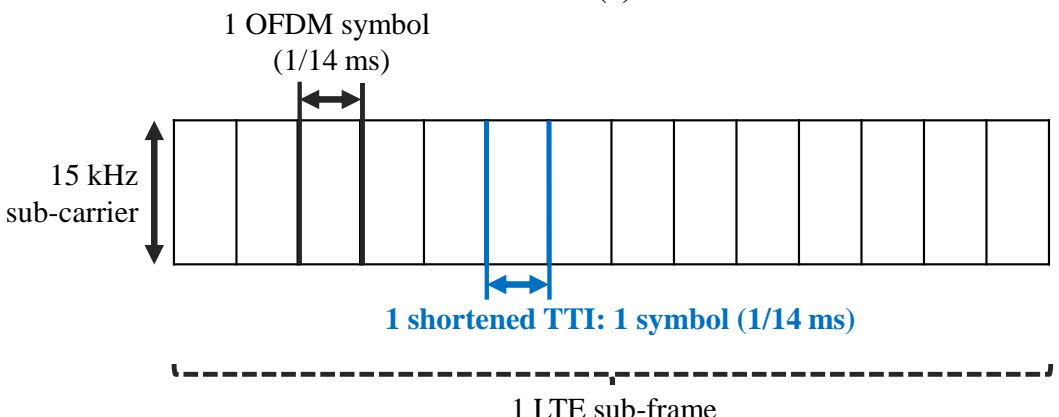

(b)

Figure 4. LTE sub-frame structure: (a) sub-frame with $1 \mathrm{~ms}$ TTI for conventional cellular systems, (b) sub-frame with shortened TTI (1/14 ms) for low latency systems.

\subsection{Cellular V2X}

Both cellular and DSRC technologies have their benefits and limitations when used for the implementation of vehicular networks. Although DSRC-based vehicular networks are widely used and are proven to be effective, they suffer from certain limitations such as channel access delay, limited coverage, and limited QoS guarantee [71]. On the other hand, existing cellular networks (UMTS and LTE) are not optimized for vehicular communication and, hence, may result in cellular network congestion in dense areas [72]. Accordingly, to 
overcome these limitations, the Third Generation Partnership Project (3GPP) published Release 14 to support vehicle-to-everything (V2X) communication with low latency and a high data rate, and referred to this evolution as LTE-V, or cellular V2X (C-V2X) [73].

The C-V2X specifications in Rel. 14 are based on existing LTE standards and, hence, can be rapidly deployed with minimum additional infrastructure costs. C-V2X supports maximum vehicle speed of $250 \mathrm{~km} / \mathrm{h}$ with a maximum relative speed of $500 \mathrm{~km} / \mathrm{h}$ [38]. It can operate using the ITS-dedicated $5.9 \mathrm{GHz}$ band with a coverage range of $450 \mathrm{~m}$ as well as in the licensed LTE frequency bands with typical LTE coverage. Nevertheless, the high relative speeds at $5.9 \mathrm{GHz}$ introduce a high Doppler shift of $\approx 2700 \mathrm{~Hz}$, leading to channel variations within the sub-frame and introducing challenges in channel estimation [74]. Accordingly, some advanced link layer enhancements are introduced in Release 14 to address these challenges, improve channel scheduling and effectively manage the resources used in V2X communications [75-77].

Two communication modes of C-V2X are introduced in Release 14: mode 3 and mode 4 . In mode 3 , the cellular base stations, i.e., LTE e-Node-B (eNB), allocate and manage resources used by the vehicles for V2X communications $[75,76]$. In mode 4 , on the other hand, distributed allocation is performed and vehicles select and manage the required resources autonomously. The authors in [78] present an analytical model for the performance of V2X in mode 4, and formulate the packet delivery ratio (PDR) as a function of the distance between the transmitter and the receiver. Furthermore, resource allocation in C-V2X where vehicles share resources with cellular users is discussed in [79-81] considering the latency constraints of V2V links.

Despite offering better resource management than DSRC, C-V2X is still quite intolerant to high density networks [73], particularly in mode 4 where interference increases with higher traffic density as the frequency re-use distance decreases. The authors in [73] compare the performance of LTE cellular V2X operating in mode 4 to DSRC technology in a highway scenario. The obtained results show that when DSRC is set to the default data rate of $6 \mathrm{Mbps}, \mathrm{C}-\mathrm{V} 2 \mathrm{X}$ provides better packet delivery ratio (PDR), whereas increasing the data rate to $18 \mathrm{Mbps}$ allows DSRC to improve its performance and achieve a higher PDR. Furthermore, the results illustrate that, similar to DSRC, as the number of transmitted packets increases, the performance of C-V2X shows significant degradation. Hence, the same problem of inefficient operation in high density charging scenarios is expected to take place using $\mathrm{C}-\mathrm{V} 2 \mathrm{X}$ communications, and further enhancements to the link capacity are required.

\section{3. $5 G N R-V 2 X$}

In addition to congestion intolerance, a major drawback of C-V2X with respect to its utilization for EV charging management is that it only provisions for broadcast communications and, hence, does not allow establishing dedicated sessions for direct exchange of vehicular information between the network entities. Accordingly, a further advanced cellular V2X network is required to address different variations of vehicular communication requirements including unicast communications to maintain the privacy of EV charging information. This has motivated the development of 5G V2X technologies that leverage on existing LTE, LTE-advanced (LTE-A) and LTE C-V2X, while fulfilling additional requirements of ultra-high reliability, high mobility, lower latency, and higher density connections [38]. This is particularly addressed in 3GPP Release 16 and is referred to as 5G New Radio (NR)-V2X.

NR-V2X technology offers several advantages over LTE C-V2X. The sub-6 GHz and mmWave frequency ranges offer a single-user bandwidth of 100 and $400 \mathrm{MHz}$, respectively, which are both significantly higher than the maximum LTE bandwidth of $20 \mathrm{MHz}$ [82]. Accordingly, the higher bandwidth and higher bandwidth efficiency, together with the smaller control overhead allows NR-V2X to offer higher data rates for vehicular communications in comparison with C-V2X [58]. In addition, NR-V2X allows operation in modes 1 and 2, both of which allow EVs to communicate directly with one another. The difference 
between the two modes is in the resource allocation, which is performed by the 5G base station (gNodeB) in mode 1 but is autonomously performed in mode 2 allowing device-todevice (D2D) vehicular communication [57]. Nevertheless, the key distinguishing feature of NR-V2X for enabling EV charging coordination and management is the support for unicast, groupcast, and broadcast messages [56,57]. In this way, an EV user can initiate multiple dedicated communication sessions with the charging infrastructure, an energy sharing vehicle or other neighboring vehicles without overloading other network entities.

The technical specifications of DSRC, LTE C-V2X, and 5G NR-V2X are summarized and compared in Tables 3 and 4.

Table 3. Comparison between specifications of IEEE 802.11p and IEEE 802.11bd DSRC [56].

\begin{tabular}{lll}
\hline Parameters & IEEE 802.11p DSRC & IEEE 802.11bd DSRC \\
\hline Frequency bands & $5.9 \mathrm{GHz}$ & $5.9 \mathrm{GHz} \& 60 \mathrm{GHz}$ \\
Subcarrier spacing & $156.25 \mathrm{kHz}$ & $78.125 \mathrm{kHz}, 156.25 \mathrm{kHz} \& 312.5 \mathrm{kHz}$ \\
Retransmissions & None & Congestion dependent \\
Waveform & OFDM with BCC & OFDM with LDPC \\
Modulation scheme & QPSK & QPSK, 16-QAM \\
Communication types & Broadcast & Broadcast \\
\hline
\end{tabular}

Table 4. Comparison between specifications of LTE C-V2X and 5G NR-V2X [56].

\begin{tabular}{lll}
\hline Parameters & Rel.14 C-V2X & Rel.16 NR-V2X \\
\hline Frequency bands & LTE bands & sub-6 GHz and mmWave \\
Subcarrier spacing & $15 \mathrm{kHz}$ & $\begin{array}{l}\text { sub-6 GHz: 15, 30, and 60 kHz } \\
\end{array}$ \\
$\begin{array}{ll}\text { Retransmissions } \\
\text { Waveform }\end{array}$ & Blind & HARQ $60,120 \mathrm{kHz}$ \\
$\begin{array}{l}\text { Modulation scheme } \\
\text { Communication types }\end{array}$ & QPSK, 16-QAM & OFDM \\
\hline
\end{tabular}

\section{5G Technology Integration}

In order to satisfy the different requirements of vehicular networks, a multi-tier heterogeneous network that integrates different vehicular communication technologies is recommended by $[50,83-85]$ such that it integrates different network technologies including mmWavs, D2D [86], massive MIMO [87,88], and software-defined networks (SDN) [89] to meet the communication requirements of different use cases. In these hybrid networks, the architecture can be arranged in a hierarchical or flat manner. In hierarchical architectures, each entity in the network belongs to a hierarchical level, and a specific technology is used for communication between the entities in different levels and a group of entities at the same level $[90,91]$. Alternatively, in flat architectures, the communication between the nodes is selected based on the type of data being transmitted or other performance metrics [92,93]. Accordingly, a multi-tier 5G network is proposed in [94] to support vehicular communication, and the proposed network model is a HetNet that incorporates D2D communication, mmWaves, and a cloud platform.

mmWave frequencies provide gigabits-per-second (Gbps) data rates for vehicular communication links and allow efficient spectrum utilization for both V2V and V2I communications [95]. The small wavelengths at mmWave frequencies enable a large number of antennas to be packed in a small form factor, forming a massive MIMO communication network [96]. This generates multiple highly directional beams that compensate for the severe propagation losses at mmWave frequencies while offering the Gbps data rates. In addition, SDNs offer new network architectures with flexible programming and powerful network control capabilities. Due to these characteristics, some researchers suggested integrating SDNs into vehicular ad hoc networks (VANET) such that the SDN controller can optimize 
vehicle routing decisions, spectrum management, and network research management [97]. Furthermore, other researchers propose a vehicular-SDN (SDN-V) architecture [98] that provides better performance compared to conventional routing protocols of VANET in terms of PDR, delay, and network throughput.

With the massive data exchange expected in an IoEV with different charging modes and requirements, quasi- and non- orthogonal multiple access (NOMA) techniques are proposed to meet the demand of spectrally efficient $5 \mathrm{G}$ vehicular communications in high density networks $[99,100]$. This is because NOMA allows each resource block to be simultaneously utilized by multiple users, thereby enabling more vehicular connectivity. The authors in [101] propose a cooperative, relay-assisted NOMA to mitigate traffic congestion and reduce latency in C-V2X communications, particularly for broadcast and multicast messages. The term cooperative means that users closer to the RSUs and/or base stations act as decode-and-forward relay (DFR) to help other users with less favorable channel conditions. This is also addressed in [102,103].

SDN is also an effective enabler of IoEV, where the intelligence of the EVs can be utilized by the SDN controllers to optimize different vehicular decisions, including optimal EV routing to charging stations, EV charging scheduling, load balancing, etc. On the other hand, the authors in [91] show the effectiveness of a heterogeneous network that utilizes DSRC to support V2V communication and 5G mmWave for V2I communication, aiming to manage the required vehicular connectivity and optimize the selection of the different radios. With these additional capabilities, heterogeneous networks of different architectures are expected to address the varying requirements of different EV charging modes while catering to the expected data congestion, payload variations and latency requirements.

\section{Physical Layer Security}

Due to the broadcast nature of wireless vehicular communication networks, particularly those utilized for enabling EV charging services, several security and privacy concerns are raised due to network vulnerability to various types of threats. These include eavesdropping, jamming, and spoofing attacks, as illustrated in Figure 5.

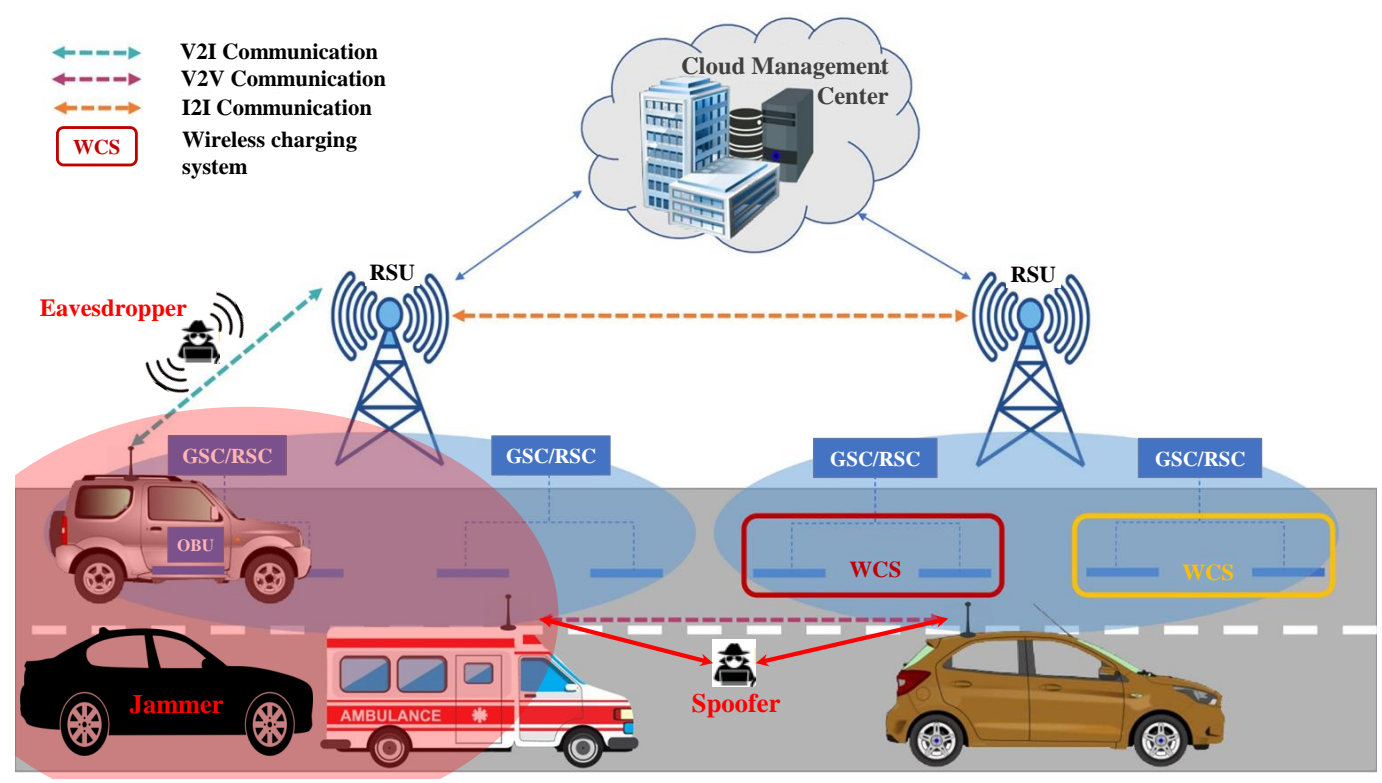

Figure 5. Overview of the different physical layer security threats in a vehicular communication network.

As shown in Figure 5, eavesdropping occurs when an illegitimate entity, i.e., an eavesdropper, listens to the messages exchanged over the communication link between two legitimate nodes, typically between the vehicle and the infrastructure. A spoofer, on 
the other hand, not only listens to the wirelessly exchanged messages but also controls the communication channel between the legitimate users. Jamming attacks at the physical layer are analogous to denial-of-service (DoS) attacks at the application layer, where a jammer generates an intentional interference to disrupt and overload the communication channel between the vehicles, leading to throughput degradation and possibly broken connectivity.

One of the most crucial requirements in vehicular communication is the security and privacy of the information shared between EVs and other network entities, to ensure protection against these different types of attacks. In wireless communications, security techniques can be categorized into: cryptographic methods and physical layer security (PLS) methods. Cryptographic methods are key-based methods that are typically applied at higher network layers to encrypt messages before transmitting them onto the wireless channel [104-106]. Nevertheless, with the high density and high mobility of vehicular networks and the heterogeneous network deployments discussed earlier, the management of different cryptographic keys becomes a challenging task [42,107]. Accordingly, physical layer security (PLS) is gaining increasing interest, as it utilizes the inherent properties of the wireless channels, namely channel fading, noise, and interference to hide the content of messages exchanged over the wireless communication channel, thereby providing security to the users [43]. PLS techniques are also more suitable for delay-sensitive applications such as DWC systems, since they are typically implemented by simple, lightweight signal processing algorithms.

In order to counteract eavesdropping attacks at the physical layer, channel-based adaptation techniques are adopted, in which the transmission parameters are adjusted to the channel characteristics of the receiver. These include beamforming, adaptive modulation, and adaptive power allocation [43]. mmWave beamforming is particularly useful to protect the channel against eavesdroppers, as it allocates narrow mmWave beams to the different legitimate users to degrade eavesdropping channels [108]. Furthermore, the integration of PLS with NOMA is considered in [107] as an approach to achieve secure spectrum sharing and protection against eavesdroppers. In addition, to avoid spoofing attacks, reciprocal channel properties such as channel state information (CSI) and received signal strength (RSS) can be utilized to authenticate the communication nodes [109]. Nevertheless, the high mobility of vehicular communications introduces rapid temporal variations to the communication channels, which increases the signalling overhead required to exchange CSI and other channel properties [96]. More effective resource allocation designs are hence required to acknowledge rapid channel variations while ensuring reliable information exchange over $\mathrm{V} 2 \mathrm{~V}$ communication links and providing protection against eavesdroppers and spoofing attacks.

MIMO and spectral spreading techniques are the most commonly adopted PLS strategies to overcome jamming [88,110]. In fact, frequency hopping spread spectrum (FHSS) is a traditional anti-jamming technique that makes the signal more resilient to jamming by spreading its frequency onto a much wider bandwidth, thereby allowing the original signal to 'hop away' from the jammed spectrum [111]. Alternatively, MIMO interference cancellation techniques cancel out jamming signals by treating them as noise while adjusting the legitimate signal direction to optimize the reception and decoding performance [111]. In $[112,113]$, jamming signals are proposed to be utilized as artificial noise (AN) that disturbs the reception of eavesdroppers and enhances the physical layer security of legitimate transmissions. Collectively, the different PLS techniques discussed in this section can be effectively employed to enhance the security of vehicular communications, particularly for EV charging coordination and management, while acknowledging the rapid temporal channel variations due to vehicular mobility.

\section{Communication in EV Charging Coordination}

Charging coordination is a key factor that contributes to the massive adoption of electric vehicles by allowing EV energy demand/supply management and resource allocation. 
The utilization of the charging energy can be improved by effective coordination of the charging plans. Usually, it is difficult to obtain an optimal charging decisions that satisfies the EV users' demand while achieving load balancing. This is because utility providers aim to maximize their profit under energy constraints, while EV users aim to maximize their received energy and QoS with minimum cost. In addition, minimizing the delay in the information exchange process is essential to ensure that EVs receive the latest scheduling information within the dynamic network changes [114].

Charging coordination strategies can be divided into two main categories: stationary charging coordination and mobility-aware charging coordination. Stationary coordination is mainly concerned with the load balancing of vehicles that are parked at home networks or public parking areas [115] and, hence, the mobility of the vehicle is ignored. Mobility-aware coordination, on the other hand, acknowledges the temporal factor of the EV motion in addition to the spatial and energy factors, by including details of the arrival/departure times to/from the charging points as well as the trip destination and the energy consumption for traveling toward the charging points $[36,116,117]$. This coordination of on-the-move EVs is essential to address EV charging demands to acknowledge both the charging time and the queuing time at the charging stations. It is also crucial in coordinating the motion of EVs toward the nearest wireless charging lane(s) in DWC systems to address urgent demands of EVs with relatively lower SoCs and provide EV drivers with a better quality-of-experience (QoE) [36].

Charging coordination can also be classified into centralized and decentralized coordination $[118,119]$. In centralized scheduling for plug-in charging stations (CS), a centralized controller makes charging decisions based on the statuses of the CSs and the EVs, which are received through reliable communication channels [120-122]. In decentralized/distributed coordination, on the other hand, each vehicle individually selects a suitable charging station based on the available information [123]. For both cases, the information should be efficiently exchanged between the charging stations and the EVs for optimal decisions. Centralized coordination is particularly useful in mobility-aware charging coordination in which EVs need to communicate with the public charging infrastructure to regulate the charging process [36]. For dynamic and quasi-dynamic EV charging systems, centralized mobility-aware scheduling also helps in defining the switching sequences of the charging pads at different charging lane locations to optimize the power transfer process [124]. Nevertheless, while centralized coordination ensures global optimal solutions due to the overall network visibility of the controller, centralized management raises privacy concerns as the participating EVs need to share real-time location updates and other information with the centralized controller for optimum decisions. This highlights the importance of implementing different physical layer security techniques, as described in Section 6, as well as application layer cryptographic techniques [104-106].

Different charging coordination and routing optimization techniques have been addressed in the literature $[72,117,122,125,126]$, together with proposals for coordinating the communication and energy exchange with mobile energy disseminators (MEDs) [18,19]. A semi-distributed V2V charging scheme is also discussed in [92], in which the charging price is determined by a central device, while the charging decisions are made by vehicles. Another semi-centralized V2V charging navigation strategy is proposed in [127], in which EVs are augmented with mobile edge computing (MEC) capabilities to adaptively choose their optimal route based on different traffic models and other information received through vehicular communications.

Nevertheless, the specifications of the underlying vehicular communication networks has received less attention in the literature, with most of the published research assuming an effective vehicular communication infrastructure through which coordination information is exchanged [119]. In addition, previously discussed papers on EVs charge scheduling and coordination mainly focus on static wired and wireless charging while only a few papers consider charge management in dynamic charging scenarios. In this section, the authors review the existing literature on the utilization of the different communication technologies 
to facilitate mobility-aware charging coordination for both stationary and dynamic EV charging systems.

\subsection{DSRC in Charging Coordination}

The authors in [72] adopt DSRC to facilitate the communication between EVs and charging stations, and to enable gathering and dissemination of information in a realtime manner using centralized coordination. The current status of the power system and real-time information of the EVs are all transmitted to a traffic server through multi-hop $\mathrm{V} 2 \mathrm{~V}$ communication. Then, the traffic sever executes an optimal charging strategy that maximizes the charging energy while minimizing the traveling cost and avoiding system overloading. Finally, charging decisions are forwarded to the EVs through the RSUs or neighboring EVs.

Another centralized coordination scheme based on DSRC is proposed by the authors in [128], aiming to minimize the total trip time considering traffic conditions. When the battery level is below a predefined threshold, an EV sends a service request to the RSU, including message ID, SoC, vehicle ID, and the current location. Afterwards, the optimal CS selection algorithm that selects the CS with the shortest service time is performed by an electro-mobility management server (EMMS), and responds to the EV by sending a service response message providing information about the selected station. Instead of single-hop communication, the authors adopted a multihop multimetric routing scheme for more efficient charging management to forward charge requests and reservation messages between EVs and EMMS. This scheme selects the next forwarding node with five metrics: distance to destination, vehicle density, vehicle trajectory, MAC losses, and available bandwidth. The forwarding scheme reduces the packet losses and the average packet delay.

The authors in [129] propose a communication framework based on DSRC for gathering real-time information about parking situations, reservations of mobile EVs, and V2V charging/discharging matching decisions. They enable relay nodes to achieve efficient wireless connection by means of V2V communications. The authors also introduce a semi-distributed coordinated charging system in which each EV decides the best charging/discharging place, while a central device performs optimal EV pair assignment. Hence, in comparison with fully centralized and fully distributed charging management schemes, a semi-centralized scheme can reduce communication congestion and computational complexity while preserving EV user privacy, improving scalability and robustness of charging, and achieving global $\mathrm{V} 2 \mathrm{~V}$ charging optimization.

The authors in $[130,131]$ also studied the use of DSRC networks in demand-side management (DSM) for wireless dynamic charging to guarantee the balance between the total demand and supply. Communication between the EV and the charging infrastructure is required to exchange information such as a congestion signal, pricing information, and charging profile. DSRC is implemented to enable information exchange, where the RSU is placed in the middle of the charging lane, and sends a congestion signal to EVs indicating that the charging demand exceeds the supply to reduce the charging rate.

\subsection{Cellular Technologies in Charging Coordination}

Cellular networks are generally selected for enabling centralized coordination due to their ubiquitous communication range and high reliability of the physical link. Considering LTE as the communication network between a group of charging stations and EV aggregators, the authors in [132] study the effect of communication delay on the performance of EV aggregators. In their simulations, the communication delay is defined as the time from the arrival of a message from the control center to an aggregator until the EV receives the scheduling message. Accordingly, the authors in [132] consider queuing and transmission delays assuming that scheduling and processing delays are negligible. Their results reveal that the communication delay is approximately constant when the number of charging points is less than 300 , beyond which the delay encounters an exponential increase as the number of EVSEs increases, and the power system becomes unstable. This 
large delay in high density scenarios reduces the efficiency of the coordination process and negatively impacts the user's QoE, particularly for users with urgent requirements such as low SoC, high demand, etc. This accordingly motivates the utilization of 5G NR-V2X communications for EV charging coordination. The ultra-low latency, high reliability, and high capacity of this technology provides robust operation in high density scenarios and improves the QoE encountered by the users.

In addition to the high density operation, large network capacity, and significantly high throughput are other desirable characteristics that motivate the use of NR-V2X for charging coordination. This is because, in an IoEV with intelligent vehicles and extensive data generated from different on-board and off-board sensors, effective coordination of the charging process involves massive V2V, V2I, and I2V data exchange, demanding significantly higher data rates and little delay tolerance. Reliability requirements as high as $99.9 \%$ are also desired to guarantee the accuracy of the charging information exchanged between the different network entities and to avoid unfavorable impacts on the energy grid due to inefficient charging coordination $[27,133]$. A comprehensive radio interface design based on $5 \mathrm{G}$ technologies, named 5GCar, is proposed in $[134,135]$ to address these requirements by integrating different technology components to meet the stringent ultra-low latency, ultra-high reliability, and high connection density requirements of $\mathrm{V} 2 \mathrm{X}$ communications.

For DWC systems, charging coordination involves coordinating the activation of charging lanes sections, optimizing the vehicle routes to the nearest charging lanes, and prioritizing low energy vehicles to receive a larger amount of power from the corresponding charging lanes. The authors in [136] discuss wireless dynamic charging in a highway scenario, taking into account the vehicle's motion and battery characteristics while considering V2I communication for information exchange.

\section{Conclusions}

Large-scale adoption of electric vehicles is expected to reduce environmental pollution, yet is hindered by the range anxiety of EV drivers due to the limited driving range, long recharging time, and low penetration of fast recharging facilities in addition to the high purchase prices of commercial EVs. Wireless EV charging solutions offer convenient and reliable charging with no physical connectivity between the EV and the charging points, thereby enabling dynamic on-the-road EV charging with minimal charging downtime and reduced range anxiety.

Nevertheless, in order to achieve efficient charging, information on traffic conditions, battery level, waiting time, and charging system state must be exchanged between the EVs and the charging infrastructure. Therefore, reliable communication links between EVs and the charging systems are necessary to exchange information, monitor system states, and coordinate charging decisions. High throughput, low latency, and high security are other desirable characteristics of the vehicular communication links to guarantee the desired QoS. This paper reviewed the existing literature on vehicular communication technologies and their utilization for static and dynamic EV charging coordination and management. The radio access technologies for vehicular communication were presented and the communication requirements for EV charging services were highlighted. Different physical layer security techniques were also surveyed to address the security requirements of vehicular communications. The role of vehicular communication technologies in EV charging management and coordination was then discussed in detail.

Among the different radio access technologies for vehicular communications surveyed in this work, LTE cellular networks offer the advantages of ubiquitous coverage, high QoS, and infrastructure readiness in comparison with the DSRC networks proposed for VANETs. Nevertheless, LTE networks are not optimized for vehicular communications and, hence, suffer from inefficient operation in high density scenarios. The same issue persists with LTE C-V2X networks despite their improved mobility support, which accordingly impacts EV charging coordination in locations with high traffic density and high EV charging demands. In contrast, 5G NR-V2X communication networks offer improved performance in high 
dense networks while providing ultra-low latency, high reliability, and high mobility support. Nevertheless, in order to satisfy the requirements of different EV charging modes in different traffic density levels and network congestion scenarios, heterogeneous vehicular communication networks need to be deployed to utilize technologies such as massive MIMO, mmWave, and SDNs to enable effective EV charging coordination. Furthermore, with this massive information exchange between different entities at high vehicular mobility levels, advanced physical layer security techniques need to be implemented to protect the communication channels from illegitimate attacks.

Collectively, the evolution of different vehicular RATs and different PLS strategies is expected to offer more efficient mobility-aware coordination and scheduling and better energy management. Nevertheless, most of the published literature on EV charging coordination assumes the existence of an underlying effective vehicular communication infrastructure, without studying the specific requirements of the communication network for effective EV charging management in an IoEV. Hence, further studies are required to develop the end-to-end architecture of the IoEV, by defining the roles of the different RATs and PLS techniques for effective EV charging coordination.

Author Contributions: Conceptualization, E.E., I.A., M.H. and A.O.; methodology, I.A. and E.E.; software, I.A.; validation, M.H. and A.O.; formal analysis, E.E. and I.A.; investigation, I.A. and E.E.; resources, M.H. and A.O.; data curation, I.A.; writing-original draft preparation, E.E.; writingreview and editing, M.H. and A.O.; visualization, E.E., I.A., M.H. and A.O.; supervision, M.H. and A.O.; project administration, M.H. and A.O.; funding acquisition, M.H. All authors have read and agreed to the published version of the manuscript.

Funding: This work is jointly supported by the American University of Sharjah through SCRI grant number SCRI 18-CEN-10, and by Sharjah Research Academy (SRA), Sharjah, United Arab Emirates.

Conflicts of Interest: The authors declare no conflict of interest.

\section{References}

1. He, Y.; Venkatesh, B.; Guan, L. Optimal scheduling for charging and discharging of electric vehicles. IEEE Trans. Smart Grid 2012, 3, 1095-1105. [CrossRef]

2. Patil, D.; McDonough, M.K.; Miller, J.M.; Fahimi, B.; Balsara, P.T. Wireless Power Transfer for Vehicular Applications: Overview and Challenges. IEEE Trans. Transp. Electrif. 2018, 4, 3-37. [CrossRef]

3. Habib, S.; Khan, M.M.; Abbas, F.; Sang, L.; Shahid, M.U.; Tang, H. A Comprehensive Study of Implemented International Standards, Technical Challenges, Impacts and Prospects for Electric Vehicles. IEEE Access 2018, 6, 13866-13890. [CrossRef]

4. Desreveaux, A.; Hittinger, E.; Bouscayrol, A.; Castex, E.; Sirbu, G.M. Techno-Economic Comparison of Total Cost of Ownership of Electric and Diesel Vehicles. IEEE Access 2020, 8, 195752-195762. [CrossRef]

5. Institution of Engineering and Technology (IET). What Is a Smart Grid? 2013. Available online: https://www.theiet.org/media/ 1251/smart-grids.pdf (accessed on 1 June 2021).

6. Javaid, N.; Hafeez, G.; Iqbal, S.; Alrajeh, N.; Alabed, M.S.; Guizani, M. Energy Efficient Integration of Renewable Energy Sources in the Smart Grid for Demand Side Management. IEEE Access 2018, 6, 77077-77096. [CrossRef]

7. Mude, K.N.; Dashora, H.K.; Bertoluzzo, M.; Buja, G. From wired to in-moving charging of the electric vehicles. In Proceedings of the WSEAS International Conference on Development, Energy, Environment, Economics (DEEE), Lisbon, Portugal, 30 October-1 November 2014; pp. 33-42.

8. Ahmad, A.; Alam, M.S.; Chabaan, R. A Comprehensive Review of Wireless Charging Technologies for Electric Vehicles. IEEE Trans. Transp. Electrif. 2018, 4, 38-63. [CrossRef]

9. Electric Power Research Institute. Interoperability of Public Electric Vehicle Charging Infrastructure. 2019. Available online: https: // www.eei.org/issuesandpolicy/electrictransportation/Documents/Final\%20Joint\%20Interoperability\%20Paper.pdf (accessed on 20 June 2021).

10. Jang, Y.J. Survey of the operation and system study on wireless charging electric vehicle systems. Transp. Res. Part C Emerg. Technol. 2018, 95, 844-866. [CrossRef]

11. Panchal, C.; Stegen, S.; Lu, J. Review of static and dynamic wireless electric vehicle charging system. J. Eng. Sci. Technol. 2018, 21, 922-937. [CrossRef]

12. ElGhanam, E.; Hassan, M.; Osman, A. Design of a High Power, LCC-Compensated, Dynamic, Wireless Electric Vehicle Charging System with Improved Misalignment Tolerance. Energies 2021, 14, 885. [CrossRef]

13. ElGhanam, E.A.; Hassan, M.S.; Osman, A.H. Deployment Optimization of Dynamic Wireless Electric Vehicle Charging Systems: A Review. In Proceedings of the 2020 IEEE International IOT, Electronics and Mechatronics Conference (IEMTRONICS), Vancouver, BC, Canada, 9-12 September 2020; pp. 1-7. [CrossRef] 
14. Mohamed, A.A.; Lashway, C.R.; Mohammed, O. Modeling and feasibility analysis of quasi-dynamic WPT system for EV applications. IEEE Trans. Transp. Electrif. 2017, 3, 343-353. [CrossRef]

15. Liu, Z.; Song, Z. Robust planning of dynamic wireless charging infrastructure for battery electric buses. Transp. Res. Part C Emerg. Technol. 2017, 83, 77-103. [CrossRef]

16. Jeong, S.; Jang, Y.J.; Kum, D. Economic Analysis of the Dynamic Charging Electric Vehicle. IEEE Trans. Power Electron. 2015, 30, 6368-6377. [CrossRef]

17. Jang, Y.J.; Suh, E.S.; Kim, J.W. System Architecture and Mathematical Models of Electric Transit Bus System Utilizing Wireless Power Transfer Technology. IEEE Syst. J. 2016, 10, 495-506. [CrossRef]

18. Kosmanos, D.; Maglaras, L.A.; Mavrovouniotis, M.; Moschoyiannis, S.; Argyriou, A.; Maglaras, A.; Janicke, H. Route Optimization of Electric Vehicles Based on Dynamic Wireless Charging. IEEE Access 2018, 6, 42551-42565. [CrossRef]

19. Maglaras, L.; Jiang, J.; Maglaras, A.; Topalis, F.; Moschoyiannis, S. Dynamic wireless charging of electric vehicles on the move with Mobile Energy Disseminators. Int. J. Adv. Comput. Sci. Appl. 2015, 6, 239-251. [CrossRef]

20. Mou, X.; Zhao, R.; Gladwin, D.T. Vehicle to Vehicle Charging (V2V) Bases on Wireless Power Transfer Technology. In Proceedings of the IECON 2018-44th Annual Conference of the IEEE Industrial Electronics Society, Washington, DC, USA, 21-23 October 2018; pp. 4862-4867.

21. Zhang, R.; Zhang, S.; Qian, Z.; Xiao, M.; Wu, J.; Ge, J.; Lu, S. Collaborative Interactive Wireless Charging in a Cyclic Mobispace. In Proceedings of the 2018 IEEE/ACM 26th International Symposium on Quality of Service (IWQoS), Banff, AB, Canada, 4-6 June 2018; pp. 1-10.

22. Shen, X.; Fantacci, R.; Chen, S. Internet of Vehicles [Scanning the Issue]. Proc. IEEE 2020, 108, 242-245. [CrossRef]

23. Bayram, I.S.; Papapanagiotou, I. A survey on communication technologies and requirements for internet of electric vehicles. EURASIP J. Wirel. Commun. Netw. 2014, 223, 1-8. [CrossRef]

24. Gil, A.; Sauras-Perez, P.; Taiber, J. Communication requirements for dynamic wireless power transfer for battery electric vehicles. In Proceedings of the 2014 IEEE International Electric Vehicle Conference (IEVC), Florence, Italy, 17-19 December 2014; pp. 1-7.

25. Smiai, O.; Bellotti, F.; De Gloria, A.; Berta, R.; Amditis, A.; Damousis, Y.; Winder, A. Information and communication technology research opportunities in dynamic charging for electric vehicle. In Proceedings of the 2015 Euromicro Conference on Digital System Design, Madeira, Portugal, 26-28 August 2015; pp. 297-300.

26. U.S. Department of Energy. Communications Requirements of Smart Grid Technologies. 2010. Available online: https://www. energy.gov/sites/default/files/gcprod/documents/Smart_Grid_Communications_Requirements_Report_10-05-2010.pdf (accessed on 1 June 2021).

27. Kuzlu, M.; Pipattanasomporn, M.; Rahman, S. Communication network requirements for major smart grid applications in HAN, NAN and WAN. Comput. Netw. 2014, 67, 74-88. [CrossRef]

28. Al-Anbagi, I.; Mouftah, H.T. WAVE 4 V2G: Wireless access in vehicular environments for vehicle-to-grid applications. Veh. Commun. 2016, 3, 31-42. [CrossRef]

29. MacHardy, Z.; Khan, A.; Obana, K.; Iwashina, S. V2X Access Technologies: Regulation, Research, and Remaining Challenges. IEEE Commun. Surv. Tutor. 2018, 20, 1858-1877. [CrossRef]

30. Rinaldi, S.; Pasetti, M.; Sisinni, E.; Bonafini, F.; Ferrari, P.; Rizzi, M.; Flammini, A. On the mobile communication requirements for the demand-side management of electric vehicles. Energies 2018, 11, 1220. [CrossRef]

31. Lee, M.; Newman, R.; Latchman, H.; Katar, S.; Yonge, L. HomePlug 1.0 powerline communication LANs-Protocol description and performance results. Int. J. Commun. Syst. 2003, 16, 447-473. [CrossRef]

32. Su, W.; Eichi, H.; Zeng, W.; Chow, M. A Survey on the Electrification of Transportation in a Smart Grid Environment. IEEE Trans. Ind. Inform. 2012, 8, 1-10. [CrossRef]

33. Baker, N. ZigBee and Bluetooth strengths and weaknesses for industrial applications. Comput. Control Eng. J. 2005, 16, 20-25. [CrossRef]

34. Su, W.; Zeng, W.; Chow, M. A digital testbed for a PHEV/PEV enabled parking lot in a Smart Grid environment. In Proceedings of the 2012 IEEE PES Innovative Smart Grid Technologies (ISGT), Washington, DC, USA, 16-20 January 2012; pp. 1-7.

35. Conti, M.; Fedeli, D.; Virgulti, M. B4V2G: Bluetooth for electric vehicle to smart grid connection. In Proceedings of the 2011-Ninth International Workshop on Intelligent Solutions in Embedded Systems, Regensburg, Germany, 7-8 July 2011; pp. 13-18.

36. Cao, Y.; Jiang, T.; Kaiwartya, O.; Sun, H.; Zhou, H.; Wang, R. Toward Pre-Empted EV Charging Recommendation Through V2V-Based Reservation System. IEEE Trans. Syst. Man Cybern. Syst. 2021, 51, 3026-3039. [CrossRef]

37. Bwalya, K. Protocols and Design Structures for Vehicular Networks. In Connected Vehicles in the Internet of Things: Concepts, Technologies and Frameworks for the IoV; Zaigham, M., Ed.; Springer International Publishing: Cham, Switzerland, 2020; Chapter 5, pp. 101-119.

38. Singh, P.; Singh, R.; Nandi, S.; Ghafoor, K.; Nandi, S. Seamless V2I Communication in HetNet: State-of-the-Art and Future Research Directions. In Connected Vehicles in the Internet of Things: Concepts, Technologies and Frameworks for the IoV; Zaigham, M., Ed.; Springer International Publishing: Cham, Switzerland, 2020; Chapter 3, pp. 37-83.

39. He, R.; Molisch, A.F.; Tufvesson, F.; Zhong, Z.; Ai, B.; Zhang, T. Vehicle-to-Vehicle Propagation Models With Large Vehicle Obstructions. IEEE Trans. Intell. Transp. Syst. 2014, 15, 2237-2248. [CrossRef] 
40. La, V.H.; Cavalli, A. Security Attacks and Solutions in Vehicular Ad Hoc Networks: A Survey. Int. J. Adhoc Netw. Syst. 2014, 4,1-20. [CrossRef]

41. Vijayakumar, P.; Azees, M.; Kannan, A.; Jegatha Deborah, L. Dual Authentication and Key Management Techniques for Secure Data Transmission in Vehicular Ad Hoc Networks. IEEE Trans. Intell. Transp. Syst. 2016, 17, 1015-1028. [CrossRef]

42. Furqan, H.M.; Solaija, M.S.J.; Hamamreh, J.M.; Arslan, H. Intelligent Physical Layer Security Approach for V2X Communication. arXiv 2020, arXiv:1905.05075.

43. Hamamreh, J.M.; Furqan, H.M.; Arslan, H. Classifications and Applications of Physical Layer Security Techniques for Confidentiality: A Comprehensive Survey. IEEE Commun. Surv. Tutor. 2019, 21, 1773-1828. [CrossRef]

44. Han, W.; Xiao, Y. Privacy preservation for V2G networks in smart grid: A survey. Comput. Commun. 2016, 91, 17-28. [CrossRef]

45. Rezgui, J.; Cherkaoui, S.; Said, D. A two-way communication scheme for vehicles charging control in the smart grid. In Proceedings of the 2012 8th International Wireless Communications and Mobile Computing Conference (IWCMC), Limassol, Cyprus, 27-31 August 2012; pp. 883-888.

46. Araniti, G.; Campolo, C.; Condoluci, M.; Iera, A.; Molinaro, A. LTE for vehicular networking: A survey. IEEE Commun. Mag. 2013, 51, 148-157. [CrossRef]

47. Fuentes, M.; Carcel, J.L.; Dietrich, C.; Yu, L.; Garro, E.; Pauli, V.; Lazarakis, F.I.; Grondalen, O.; Bulakci, M.; Yu, J.; et al. 5G New Radio Evaluation Against IMT-2020 Key Performance Indicators. IEEE Access 2020, 8, 110880-110896. [CrossRef]

48. Belanovic, P.; Valerio, D.; Paier, A.; Zemen, T.; Ricciato, F.; Mecklenbrauker, C.F. On wireless links for vehicle-to-infrastructure communications. IEEE Trans. Veh. Technol. 2009, 59, 269-282. [CrossRef]

49. Xu, Z.; Li, X.; Zhao, X.; Zhang, M.H.; Wang, Z. DSRC versus 4G-LTE for Connected Vehicle Applications: A Study on Field Experiments of Vehicular Communication Performance. J. Adv. Transp. 2017, 2017, 2750452. [CrossRef]

50. Abboud, K.; Omar, H.A.; Zhuang, W. Interworking of DSRC and Cellular Network Technologies for V2X Communications: A Survey. IEEE Trans. Veh. Technol. 2016, 65, 9457-9470. [CrossRef]

51. Mohammad, S.A.; Rasheed, A.; Qayyum, A. VANET architectures and protocol stacks: A survey. In Proceedings of the International Workshop on Communication Technologies for Vehicles, Oberpfaffenhofen, Germany, 23-24 March 2011; pp. 95-105.

52. Ahmed, S.A.; Ariffin, S.H.; Fisal, N. Overview of wireless access in vehicular environment (WAVE) protocols and standards. Indian J. Sci. Technol. 2013, 6, 4994-5001. [CrossRef]

53. Hussain, S.S.; Ustun, T.S.; Nsonga, P.; Ali, I. IEEE 1609 WAVE and IEC 61850 standard communication based integrated EV charging management in smart grids. IEEE Trans. Veh. Technol. 2018, 67, 7690-7697. [CrossRef]

54. Gil, A.; Taiber, J. A literature review in dynamic wireless power transfer for electric vehicles: Technology and infrastructure integration challenges. In Sustainable Automotive Technologies 2013; Springer International Publishing: Cham, Switzerland, 2014; pp. 289-298.

55. Uzcátegui, R.A.; De Sucre, A.J.; Acosta-Marum, G. Wave: A tutorial. IEEE Commun. Mag. 2009, 47, 126-133. [CrossRef]

56. Naik, G.; Choudhury, B.; Park, J. IEEE 802.11bd \& 5G NR V2X: Evolution of Radio Access Technologies for V2X Communications. IEEE Access 2019, 7, 70169-70184.

57. Zeadally, S.; Javed, M.A.; Hamida, E.B. Vehicular Communications for ITS: Standardization and Challenges. IEEE Commun. Stand. Mag. 2020, 4, 11-17. [CrossRef]

58. Anwar, W.; Franchi, N.; Fettweis, G. Physical Layer Evaluation of V2X Communications Technologies: 5G NR-V2X, LTE-V2X, IEEE 802.11bd, and IEEE 802.11p. In Proceedings of the 2019 IEEE 90th Vehicular Technology Conference (VTC2019-Fall), Honolulu, HI, USA, 22-25 September 2019; pp. 1-7.

59. Sharanappa, P.; Mahabaleshwar, S. Performance analysis of CSMA, MACA and MACAW protocols for VANETs. Int. J. Future Comput. Commun. 2014, 3, 129.

60. IEEE. IEEE Standard-Part 11: Wireless LAN Medium Access Control (MAC) and Physical Layer (PHY) Specifications; Standard, IEEE Std 802.11-2016; IEEE: Piscataway, NJ, USA, 2016.

61. Hadded, M.; Muhlethaler, P.; Laouiti, A.; Zagrouba, R.; Saidane, L.A. TDMA-based MAC protocols for vehicular ad hoc networks: A survey, qualitative analysis, and open research issues. IEEE Commun. Surv. Tutor. 2015, 17, 2461-2492. [CrossRef]

62. Jayaraj, V.; Hemanth, C.; Sangeetha, R. A survey on hybrid MAC protocols for vehicular ad-hoc networks. Veh. Commun. 2016, 6, 29-36. [CrossRef]

63. Nguyen, V.; Oo, T.Z.; Chuan, P.; Hong, C.S. An efficient time slot acquisition on the hybrid TDMA/CSMA multichannel MAC in VANETs. IEEE Commun. Lett. 2016, 20, 970-973. [CrossRef]

64. Torabi, N.; Ghahfarokhi, B.S. A bandwidth-efficient and fair CSMA/TDMA based multichannel MAC scheme for V2V communications. Telecommun. Syst. 2017, 64, 367-390. [CrossRef]

65. ETSI. ETSI Technical Report: Intelligent Transport Systems (ITS); Vehicular Communications; Basic Set of Applications; Definitions; Standard, ETSI TR 102638 V1.1.1; European Telecommunications Standards Institute (ETSI): Sophia Antipolis, France, 2009.

66. Sommer, C.; Schmidt, A.; Chen, Y.; German, R.; Koch, W.; Dressler, F. On the feasibility of UMTS-based traffic information systems. Ad Hoc Netw. 2010, 8, 506-517. [CrossRef]

67. Mangel, T.; Kosch, T.; Hartenstein, H. A comparison of UMTS and LTE for vehicular safety communication at intersections. In Proceedings of the 2010 IEEE Vehicular Networking Conference, Jersey City, NJ, USA, 13-15 December 2010; pp. 293-300. 
68. Abid, H.; Chung, T.C.; Lee, S.; Qaisar, S. Performance analysis of LTE smartphones-based vehicle-to-infrastrcuture communication. In Proceedings of the 2012 9th International Conference on Ubiquitous Intelligence and Computing and 9th International Conference on Autonomic and Trusted Computing, Fukuoka, Japan, 4-7 September 2012; pp. 72-78.

69. Patra, M.; Thakur, R.; Murthy, C.S.R. Improving delay and energy efficiency of vehicular networks using mobile femto access points. IEEE Trans. Veh. Technol. 2016, 66, 1496-1505. [CrossRef]

70. Lee, K.; Kim, J.; Park, Y.; Wang, H.; Hong, D. Latency of Cellular-Based V2X: Perspectives on TTI-Proportional Latency and TTI-Independent Latency. IEEE Access 2017, 5, 15800-15809. [CrossRef]

71. Liu, Z.; Lee, H.; Ali, G.; Pesch, D.; Xiao, P. A Survey on Resource Allocation in Vehicular Networks. arXiv 2019, arXiv:1909.13587.

72. Wang, M.; Liang, H.; Zhang, R.; Deng, R.; Shen, X. Mobility-aware coordinated charging for electric vehicles in VANET-enhanced smart grid. IEEE J. Sel. Areas Commun. 2014, 32, 1344-1360. [CrossRef]

73. Molina-Masegosa, R.; Gozalvez, J. LTE-V for sidelink 5G V2X vehicular communications: A new 5G technology for short-range vehicle-to-everything communications. IEEE Veh. Technol. Mag. 2017, 12, 30-39. [CrossRef]

74. Qualcomm. Cellular-V2X Technology Overview: 80-PE732-63 Rev B. Available online: https://www.qualcomm.com/media/ documents / files / c-v2x-technology-overview.pdf (accessed on 8 June 2021).

75. Fowler, S.; Häll, C.H.; Yuan, D.; Baravdish, G.; Mellouk, A. Analysis of vehicular wireless channel communication via queueing theory model. In Proceedings of the 2014 IEEE International Conference on Communications (ICC), Sydney, Australia, 10-14 June 2014; pp. 1736-1741.

76. Vinel, A. 3GPP LTE versus IEEE 802.11 p/WAVE: Which technology is able to support cooperative vehicular safety applications? IEEE Wirel. Commun. Lett. 2012, 1, 125-128. [CrossRef]

77. Nabil, A.; Kaur, K.; Dietrich, C.; Marojevic, V. Performance analysis of sensing-based semi-persistent scheduling in C-V2X networks. In Proceedings of the 2018 IEEE 88th Vehicular Technology Conference (VTC-Fall), Chicago, IL, USA, 27-30 August 2018; pp. 1-5.

78. Gonzalez-Martín, M.; Sepulcre, M.; Molina-Masegosa, R.; Gozalvez, J. Analytical models of the performance of c-v2x mode 4 vehicular communications. IEEE Trans. Veh. Technol. 2018, 68, 1155-1166. [CrossRef]

79. Guo, C.; Liang, L.; Li, G.Y. Resource allocation for low-latency vehicular communications: An effective capacity perspective. IEEE J. Sel. Areas Commun. 2019, 37, 905-917. [CrossRef]

80. Guo, C.; Liang, L.; Li, G.Y. Resource allocation for vehicular communications with low latency and high reliability. IEEE Trans. Wirel. Commun. 2019, 18, 3887-3902. [CrossRef]

81. Mei, J.; Zheng, K.; Zhao, L.; Teng, Y.; Wang, X. A latency and reliability guaranteed resource allocation scheme for LTE V2V communication systems. IEEE Trans. Wirel. Commun. 2018, 17, 3850-3860. [CrossRef]

82. 3GPP. 3GPP Technical Report: Technical Specification Group Services and System Aspects; Study on Enhancement of 3GPP Support for 5G V2X Services; Standard, 3GPP TR 22.886; 3rd Generation Partnership Project (3GPP): Sophia Antipolis Valbonne, France, 2018.

83. Zheng, K.; Zheng, Q.; Chatzimisios, P.; Xiang, W.; Zhou, Y. Heterogeneous vehicular networking: A survey on architecture, challenges, and solutions. IEEE Commun. Surv. Tutor. 2015, 17, 2377-2396. [CrossRef]

84. Zheng, Q.; Zheng, K.; Sun, L.; Leung, V.C. Dynamic performance analysis of uplink transmission in cluster-based heterogeneous vehicular networks. IEEE Trans. Veh. Technol. 2015, 64, 5584-5595. [CrossRef]

85. Dai, P.; Liu, K.; Wu, X.; Liao, Y.; Lee, V.C.S.; Son, S.H. Bandwidth efficiency and service adaptiveness oriented data dissemination in heterogeneous vehicular networks. IEEE Trans. Veh. Technol. 2018, 67, 6585-6598. [CrossRef]

86. Ahmed, I.; Ismail, M.; Hassan, M. Video Transmission Using Device-to-Device Communications: A Survey. IEEE Access 2019, 7, 131019-131038. [CrossRef]

87. Ge, X.; Cheng, H.; Mao, G.; Yang, Y.; Tu, S. Vehicular Communications for 5G Cooperative Small-Cell Networks. IEEE Trans. Veh. Technol. 2016, 65, 7882-7894. [CrossRef]

88. Busari, S.A.; Huq, K.; Mumtaz, S.; Rodriguez, J. Millimetre-wave massive MIMO for cellular vehicle-to-infrastructure communication. IET Intell. Transp. Syst. 2019, 13, 983-990. [CrossRef]

89. Chen, N.; Wang, M.; Zhang, N.; Shen, X.S.; Zhao, D. SDN-based framework for the PEV integrated smart grid. IEEE Netw. 2017, 31, 14-21. [CrossRef]

90. Liu, B.; Jia, D.; Wang, J.; Lu, K.; Wu, L. Cloud-assisted safety message dissemination in VANET-cellular heterogeneous wireless network. IEEE Syst. J. 2015, 11, 128-139. [CrossRef]

91. Sheng, Z.; Pressas, A.; Ocheri, V.; Ali, F.; Rudd, R.; Nekovee, M. Intelligent 5G vehicular networks: An integration of DSRC and mmWave communications. In Proceedings of the 2018 International Conference on Information and Communication Technology Convergence (ICTC), Jeju Island, Korea, 17-19 October 2018; pp. 571-576.

92. Wang, M.; Ismail, M.; Zhang, R.; Shen, X.; Serpedin, E.; Qaraqe, K. Spatio-Temporal Coordinated V2V Energy Swapping Strategy for Mobile PEVs. IEEE Trans. Smart Grid 2018, 9, 1566-1579. [CrossRef]

93. Bi, S.; Chen, C.; Du, R.; Guan, X. Proper handover between VANET and cellular network improves Internet access. In Proceedings of the 2014 IEEE 80th Vehicular Technology Conference (VTC2014-Fall), Vancouver, BC, Canada, 14-17 September 2014; pp. 1-5.

94. Eiza, M.H.; Ni, Q.; Shi, Q. Secure and privacy-aware cloud-assisted video reporting service in 5G-enabled vehicular networks. IEEE Trans. Veh. Technol. 2016, 65, 7868-7881. [CrossRef]

95. Dong, P.; Zheng, T.; Yu, S.; Zhang, H.; Yan, X. Enhancing vehicular communication using 5G-enabled smart collaborative networking. IEEE Wirel. Commun. 2017, 24, 72-79. [CrossRef] 
96. Liang, L.; Peng, H.; Li, G.Y.; Shen, X. Vehicular Communications: A Physical Layer Perspective. IEEE Trans. Veh. Technol. 2017, 66, 10647-10659. [CrossRef]

97. Yang, Y.; Hua, K. Emerging technologies for 5G-enabled vehicular networks. IEEE Access 2019, 7, 181117-181141. [CrossRef]

98. Correia, S.; Boukerche, A.; Meneguette, R.I. An architecture for hierarchical software-defined vehicular networks. IEEE Commun. Mag. 2017, 55, 80-86. [CrossRef]

99. Jameel, F.; Chang, Z.; Huang, J.; Ristaniemi, T. Internet of Autonomous Vehicles: Architecture, Features, and Socio-Technological Challenges. IEEE Wirel. Commun. 2019, 26, 21-29. [CrossRef]

100. Li, Q.C.; Niu, H.; Papathanassiou, A.T.; Wu, G. 5G Network Capacity: Key Elements and Technologies. IEEE Veh. Technol. Mag. 2014, 9, 71-78. [CrossRef]

101. Liu, G.; Wang, Z.; Hu, J.; Ding, Z.; Fan, P. Cooperative NOMA Broadcasting/Multicasting for Low-Latency and High-Reliability 5G Cellular V2X Communications. IEEE Internet Things J. 2019, 6, 7828-7838. [CrossRef]

102. Kim, J.B.; Lee, I.H. Capacity Analysis of Cooperative Relaying Systems Using Non-Orthogonal Multiple Access. IEEE Commun. Lett. 2015, 19, 1949-1952. [CrossRef]

103. Dahi, N.; Hamdi, N. Relaying in Non-Orthogonal Multiple Access Systems with Simultaneous Wireless Information and Power Transfer. In Proceedings of the 2018 14th International Wireless Communications Mobile Computing Conference (IWCMC), Limassol, Cyprus, 25-29 June 2018; pp. 164-168. [CrossRef]

104. Khan, A.G.; Basharat, S.; Riaz, M.U. Analysis of asymmetric cryptography in information security based on computational study to ensure confidentiality during information exchange. Int. J. Sci. Eng. Res. 2018, 992-999. [CrossRef]

105. Zhao, X.; Lin, J.; Li, H. Privacy-preserving billing scheme against free-riders for wireless charging electric vehicles. Mob. Inf. Syst. 2017, 2017, 1325698. [CrossRef]

106. Bokhari, M.U.; Shallal, Q.M. A review on symmetric key encryption techniques in cryptography. Int. J. Comput. Appl. 2016, $147,43-48$.

107. ElHalawany, B.M.; El-Banna, A.A.A.; Wu, K. Physical-Layer Security and Privacy for Vehicle-to-Everything. IEEE Commun. Mag. 2019, 57, 84-90. [CrossRef]

108. Martin-Vega, F.J.; Aguayo-Torres, M.C.; Gomez, G.; Entrambasaguas, J.T.; Duong, T.Q. Key Technologies, Modeling Approaches, and Challenges for Millimeter-Wave Vehicular Communications. IEEE Commun. Mag. 2018, 56, 28-35. [CrossRef]

109. Wang, X.; Hao, P.; Hanzo, L. Physical-layer authentication for wireless security enhancement: Current challenges and future developments. IEEE Commun. Mag. 2016, 54, 152-158. [CrossRef]

110. Kosmanos, D.; Prodromou, N.; Argyriou, A.; Maglaras, L.A.; Janicke, H. MIMO Techniques for Jamming Threat Suppression in Vehicular Networks. Mob. Inf. Syst. 2016, 2016, 8141204. [CrossRef]

111. Yan, Q.; Zeng, H.; Jiang, T.; Li, M.; Lou, W.; Hou, Y.T. Jamming Resilient Communication Using MIMO Interference Cancellation. IEEE Trans. Inf. Forensics Secur. 2016, 11, 1486-1499. [CrossRef]

112. Li, W.; Ghogho, M.; Chen, B.; Xiong, C. Secure Communication via Sending Artificial Noise by the Receiver: Outage Secrecy Capacity/Region Analysis. IEEE Commun. Lett. 2012, 16, 1628-1631. [CrossRef]

113. Zhao, N.; Yu, F.R.; Li, M.; Yan, Q.; Leung, V.C.M. Physical layer security issues in interference- alignment-based wireless networks. IEEE Commun. Mag. 2016, 54, 162-168. [CrossRef]

114. Cao, Y.; Ahmad, N.; Kaiwartya, O.; Puturs, G.; Khalid, M. Intelligent transportation systems enabled ICT framework for electric vehicle charging in smart city. In Handbook of Smart Cities; Springer International Publishing: Cham, Switzerland, 2018; pp. 311-330.

115. Dagdougui, H.; Ouammi, A.; Dessaint, L.A. Peak Load Reduction in a Smart Building Integrating Microgrid and V2B-Based Demand Response Scheme. IEEE Syst. J. 2019, 13, 3274-3282. [CrossRef]

116. Zhang, J.; Pei, Y.; Shen, J.; Wang, L.; Ding, T.; Wang, S. Charging Strategy Unifying Spatial-Temporal Coordination of Electric Vehicles. IEEE Access 2020, 8, 74853-74863. [CrossRef]

117. Ding, D.; Li, J.; Tu, P.; Wang, H.; Cao, T.; Zhang, F. Electric Vehicle Charging Warning and Path Planning Method Based on Spark. IEEE Access 2020, 8, 8543-8553. [CrossRef]

118. Mukherjee, J.C.; Gupta, A. Distributed charge scheduling of plug-in electric vehicles using inter-aggregator collaboration. IEEE Trans. Smart Grid 2016, 8, 331-341. [CrossRef]

119. Saldana, G.; San Martin, J.I.; Zamora, I.; Asensio, F.J.; Onederra, O. Electric Vehicle into the Grid: Charging Methodologies Aimed at Providing Ancillary Services Considering Battery Degradation. Energies 2019, 12, 2443. [CrossRef]

120. Liu, X.; Gao, B.; Wu, C.; Tang, Y. Demand-Side Management With Household Plug-In Electric Vehicles: A Bayesian GameTheoretic Approach. IEEE Syst. J. 2018, 12, 2894-2904. [CrossRef]

121. Luo, X.; Chan, K. Real-time scheduling of electric vehicles charging in low-voltage residential distribution systems to minimise power losses and improve voltage profile. Gener. Transm. Distrib. IET 2014, 8, 516-529. [CrossRef]

122. Wang, Y.; Su, Z.; Xu, Q.; Yang, T.; Zhang, N. A Novel Charging Scheme for Electric Vehicles With Smart Communities in Vehicular Networks. IEEE Trans. Veh. Technol. 2019, 68, 8487-8501. [CrossRef]

123. Liu, M.; Phanivong, P.K.; Callaway, D.S. Electric vehicle charging control in residential distribution network: A decentralized event-driven realization. In Proceedings of the 2017 IEEE 56th Annual Conference on Decision and Control (CDC), Melbourne, Australia, 12-15 December 2017; pp. 214-219. [CrossRef] 
124. Li, M.; Wu, X.; Zhang, Z.; Yu, G.; Wang, Y.; Ma, W. A Wireless Charging Facilities Deployment Problem Considering Optimal Traffic Delay and Energy Consumption on Signalized Arterial. IEEE Trans. Intell. Transp. Syst. 2019, 20, 4427-4438. [CrossRef]

125. Li, C.; Ding, T.; Liu, X.; Huang, C. An Electric Vehicle Routing Optimization Model With Hybrid Plug-In and Wireless Charging Systems. IEEE Access 2018, 6, 27569-27578. [CrossRef]

126. Santini, S.; Salvi, A.; Valente, A.S.; Pescape, A.; Segata, M.; Lo Cigno, R. A Consensus-Based Approach for Platooning with Intervehicular Communications and Its Validation in Realistic Scenarios. IEEE Trans. Veh. Technol. 2017, 66, 1985-1999. [CrossRef]

127. Li, G.; Sun, Q.; Boukhatem, L.; Wu, J.; Yang, J. Intelligent Vehicle-to-Vehicle Charging Navigation for Mobile Electric Vehicles via VANET-Based Communication. IEEE Access 2019, 7, 170888-170906. [CrossRef]

128. Bautista, P.B.; Cárdenas, L.L.; Aguiar, L.U.; Igartua, M.A. A traffic-aware electric vehicle charging management system for smart cities. Veh. Commun. 2019, 20, 100188.

129. Li, G.; Boukhatem, L.; Zhao, L.; Wu, J. Direct Vehicle-to-Vehicle Charging Strategy in Vehicular Ad-Hoc Networks. In Proceedings of the 2018 9th IFIP International Conference on New Technologies, Mobility and Security (NTMS), Paris, France, 26-28 February 2018; pp. 1-5.

130. Theodoropoulos, T.V.; Damousis, I.G.; Amditis, A.J. Demand-side management ICT for dynamic wireless EV charging. IEEE Trans. Ind. Electron. 2016, 63, 6623-6630. [CrossRef]

131. Theodoropoulos, T.V.; Damousis, I.G.; Portouli, E.; Amditis, A.J.; El Sayed, N.; Laporte, S. Analysis of AIMD-based DSM for EV wireless charging on the move. In Proceedings of the 2016 International Conference on Electrical Systems for Aircraft, Railway, Ship Propulsion and Road Vehicles \& International Transportation Electrification Conference (ESARS-ITEC), Toulouse, France, 2-4 November 2016; pp. 1-8.

132. Ko, K.; Sung, D.K. The effect of cellular network-based communication delays in an EV aggregator's domain on frequency regulation service. IEEE Trans. Smart Grid 2017, 10, 65-73. [CrossRef]

133. Ismail, M.; Bayram, I.; Qaraqe, K.; Serpendin, E. 5GâĂŘEnhanced Smart Grid Services. In Enabling 5G Communication Systems to Support Vertical Industries; Imran, M., Sambo, Y., Abbasi, Q., Eds.; Wiley-IEEE Press: Piscataway, NJ, USA, 2019 ; pp. 75-102.

134. Li, Z.; Fodor, G. (Eds.) Deliverable D3.1: Intermediate 5G V2X Radio; Fifth Generation Communication Automotive Research and Innovation (5GPP): Valencia, Spain, 2018.

135. Fallgren, M.; Abbas, T.; Allio, S.; Alonso-Zarate, J.; Fodor, G.; Gallo, L.; Kousaridas, A.; Li, Y.; Li, Z.; Li, Z.; et al. Multicast and Broadcast Enablers for High-Performing Cellular V2X Systems. IEEE Trans. Broadcast. 2019, 65, 454-463. [CrossRef]

136. Kong, C.; Devetsikiotis, M. Optimal charging framework for electric vehicles on the wireless charging highway. In Proceedings of the 2016 IEEE 21st International Workshop on Computer Aided Modelling and Design of Communication Links and Networks (CAMAD), Toronto, ON, Canada, 23-25 October 2016; pp. 89-94. 